\title{
Caracterización petrofísica de la formación oficina en un sector del bloque carabobo mediante métodos fractales
}

\author{
Ignacio J. Mederos V. ${ }^{1 *}$ \\ ${ }^{1}$ Laboratorio de Interpretación del subsuelo. Departamento de Geofísica, Escuela de Geología, Minas y Geofísica. \\ Universidad Central de Venezuela, Caracas, Venezuela. \\ Email: imederos30@gmail.com
}

\begin{abstract}
Resumen
El propósito es generar modelos de isopropiedades petrofísicas a partir de métodos estadísticos que contribuyan en la caracterización estática de yacimientos, el concepto de fractal el cual se define como un objeto cuya estructura se repite a diferentes escalas, fue el método aplicado. Considerando que el campo Carabobo presenta bajo ángulo, se decidió la utilización de fractales en esta investigación introduciendo como registros de entrada los de rayos gamma, densidad, resistividad y el de porosidad efectiva, a los cuales se le aplicó método para la elaboración y el análisis de modelos de isopropiedades petrofísicas, ya que cumplen con la condición de que los registros se comportan como series en tiempo, con aleatoriedad y pueden ser normalizados. A partir del análisis por la técnica de rango escalado "R/S" (re-escalamiento) se obtiene el exponente o coeficiente Hurst $(\mathrm{H})$ que indica el grado de rigurosidad y persistencia de los datos con la distancia, estableciendo de esta manera el alcance de los mismos al realizar la interpolación fractal de los pozos, generando así pseudopozos. De la integración de los pozos originales y los pseudopozos generados se estimó la distribución espacial de las propiedades petrofísicas. Corroborando que es una herramienta útil, reduce la incertidumbre en la determinación y extensión de prospectos en procesos exploratorios y mejora la visualización de las propiedades petrofísicas tanto horizontal como verticalmente.
\end{abstract}

Palabras Claves: algoritmos fractales, modelado de isopropiedades petrofísica, registros de pozos.

\section{Petrophysical characterization of oficina formation in a sector of the carabobo block by fractal methods}

\begin{abstract}
The purpose is to generate models of petrophysical isoproperties from statistical methods that contribute to the static characterization of deposits, the concept of fractal which is defined as an object whose structure is repeated at different scales, was the applied method. Considering that the Carabobo field presents low angle, it was decided to use fractals in this investigation introducing as input records those of gamma rays, density, resistivity and effective porosity, to which the method was applied. for the elaboration and analysis of petrophysical isoproperties models, since they comply with the condition that the records behave as series in time, with randomness and can be normalized. From the analysis by the scaled range technique "R/S" (reescalation), the Hurst exponent or coefficient $(\mathrm{H})$ is obtained, which indicates the degree of rigor and persistence of the data with distance, establishing in this way the scope of the same when performing the fractal interpolation of the wells, thus generating pseudopozos. The spatial distribution of the petrophysical properties was estimated from the integration of the original wells and the generated pseudo-wells. Corroborating that it is a useful tool, it reduces the uncertainty in the determination and extension of prospects in exploratory processes and improves the visualization of the petrophysical properties both horizontally and vertically.
\end{abstract}

Keywords: Fractal algorithm, models of petrophysical isoproperties wells register.

Cita: Mederos V, I. J. (2019). Caracterización petrofísica de la formación oficina en un sector del bloque carabobo mediante métodos fractales. Revista Fuentes: El reventón energético, 17(1), 71-82. 


\section{Introducción}

La caracterización de los yacimientos de hidrocarburos implementando métodos petrofísicos se han aplicado en las geociencias para ayudar a la ingeniería de yacimientos en la discriminación de las propiedades físicas de las rocas y su interacción con los fluidos. Debido a la gran extensión geográfica que se debe abarcar, resulta conveniente utilizar métodos que permitan analizar con un alto nivel de resolución y bajo costo las variaciones laterales y verticales de dichas propiedades. En la caracterización de los fluidos de yacimiento, los registros eléctricos juegan un papel muy importante, así como en la caracterización litológica los registros de rayos gamma son vitales y los registros de porosidad efectiva nos dan la información de capacidad de almacenamiento de la roca, no obstante, los elevados costos de adquisición representan un inconveniente para las compañías. Debido a esto, han surgido nuevas técnicas exploratorias a partir de métodos estadísticos (ej. redes neuronales y algoritmos fractales), siendo estas herramientas ideales para estudios de alta resolución de fluidos de yacimientos. El método de algoritmo fractal permite generar pseudopozos usando información de pozos existentes, logrando de esta forma cubrir el campo en su totalidad permitiendo mejorar la resolución en el modelado de las propiedades petrofísicas, sin necesidad de perforar nuevos pozos.
Teniendo en cuenta lo anterior el objetivo de esta investigación es analizar cualitativamente las propiedades petrofísicas extendidas en la Formación Oficina (edad Mioceno temprano a medio), ubicada en el campo Carabobo de la cuenca Oriental de Venezuela, mediante métodos estadísticos fractales generando modelos volumétricos de dichas propiedades.

\section{Marco geológico}

\section{Geología Regional}

La cuenca Oriental de Venezuela constituye la segunda cuenca petrolífera de América del sur, está situada al centro-este de Venezuela, abarcando los estados Anzoátegui, Monagas, Guárico Y Delta Amacuro. Los campos petrolíferos de esta cuenca comparten características estratigráficas y estructurales, siendo la Formación Oficina la principal roca productora (Yoris, 1997).

En donde se encuentra la Faja petrolífera del Orinoco (Fig. 1), que se subdivide en cuatro campos (Boyacá, Junín. Ayacucho y Carabobo), siendo el de interés en la investigación el campo Carabobo que se encuentra al este de la cuenca.

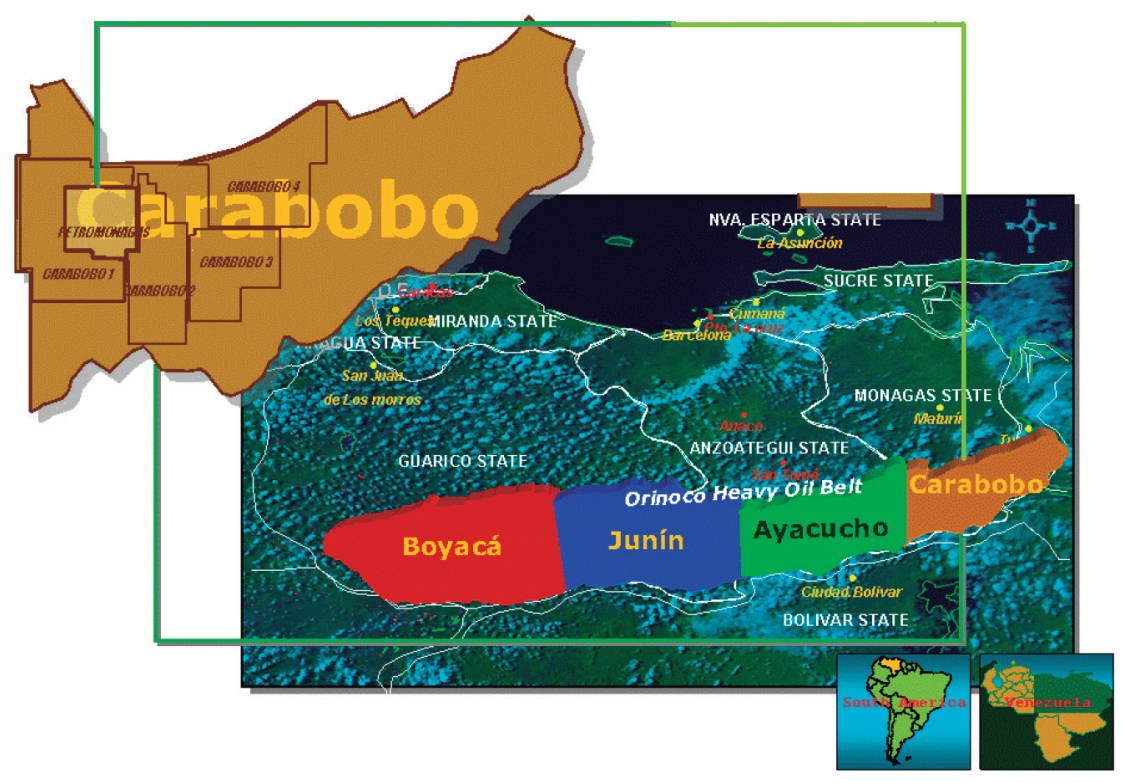

Figura 1. Mapa de ubicación del Bloque Carabobo en la Cuenca Oriental. (Tomado de Calvo, 2008).

La faja petrolífera del Orinoco corresponde al acuñamiento sur de los sedimentos terciarios de la Cuenca Oriental venezolana, por encima del basamento ígneo-metamórfico del Cratón guayanés localizado al sur del río Orinoco. En la parte más septentrional de la Faja, hacia los sectores de Carabobo y Ayacucho, los sedimentos terciarios se encuentran suprayaciendo discordantemente a una franja de poco espesor de 
sedimentos cretácicos que a su vez se adelgazan hasta llegar a desaparecer al sur (Yoris. 1997).

\section{Geología estructural Local}

Dentro del Bloque Carabobo el patrón estructural es el mismo que el resto de la Cuenca Oriental de Venezuela (Fig. 2), específicamente en la parte sur de la misma. La estructura está definida por un homoclinal de rumbo aproximado noreste-suroeste donde el buzamiento es muy suave y oscila entre 2 y 4 grados promedio. La misma está cortada por fallas normales principales de rumbo aproximado este-oeste. Estas fallas representan un importante factor de entrampamiento dentro del área de estudio, sumado al factor estratigráfico asociado a cambios laterales de facies. Esto lleva a concluir que las trampas existentes son de tipo estructural-estratigráfico, donde el componente estratigráfico es el de mayor importancia, (Yoris, 1997).



Figura 2. Rasgos estructurales de la cuenca oriental de Venezuela (Tomado de Chramcow, 2008).

Estructuralmente se observa una tendencia en el campo Carabobo de adelgazamiento hacia el este y engrosamiento hacia el oeste de los depósitos sedimentarios como se observa en la correlación estructural (Fig. 3), marcando un acuñamiento hacia el sureste. Y la mayor depositación hacia el noroeste.

\section{Geología estratigrafía Local}

La zona está constituida por las formaciones: Merecure (Oligoceno- Mioceno Temprano), Oficina (Mioceno Temprano a Medio), Freites (Mioceno Tardío), Las Piedras (Mioceno- Plioceno) y Mesa (Pleistoceno). Siendo la Formación Oficina la más importante, debido a su interés económico como reservorio, la misma es descrita como intercalaciones areniscas y lutitas. Funkhouser et al (1948) y Hedberg (1950) definen esta formación como intercalaciones de lutitas de colores grisáceos oscuro con areniscas y limolitas interestratificadas, de colores claros y granos de fino a grueso, presentando un contacto suprayacente concordante con la Formación Freites e infrayacente concordante con la Formación Merecure.

La cobertura sedimentaria de la cuenca, es característica de un ambiente fluvio-deltáico continental, con presencia de areniscas arcillosas y delgadas propias de estos ambientes. Lo que se sostiene en las afirmaciones de Crespo (2008), quien considera que la Formación Oficina se sedimentó en un inmenso complejo fluviodeltaico, donde las areniscas lenticulares y de relleno de canales de ríos son comunes, entre ellas se encuentran estructuras por arenas finas, arenas arcillosas y arenas limosas, que suelen ser idóneas para la acumulación de 
hidrocarburos. Estas estructuras lenticulares presentan pocos pies de espesor, de aproximadamente 150 a 250 pies Banks (1958), mientras que las arenas laminares o arcillas pueden tener varios pies de espesor, entre 656 pies a 1148 pies de largo y poseen variación lateral en el tamaño de grano desde arenas en un extremo hasta arcillas en el otro Busnego (2012).

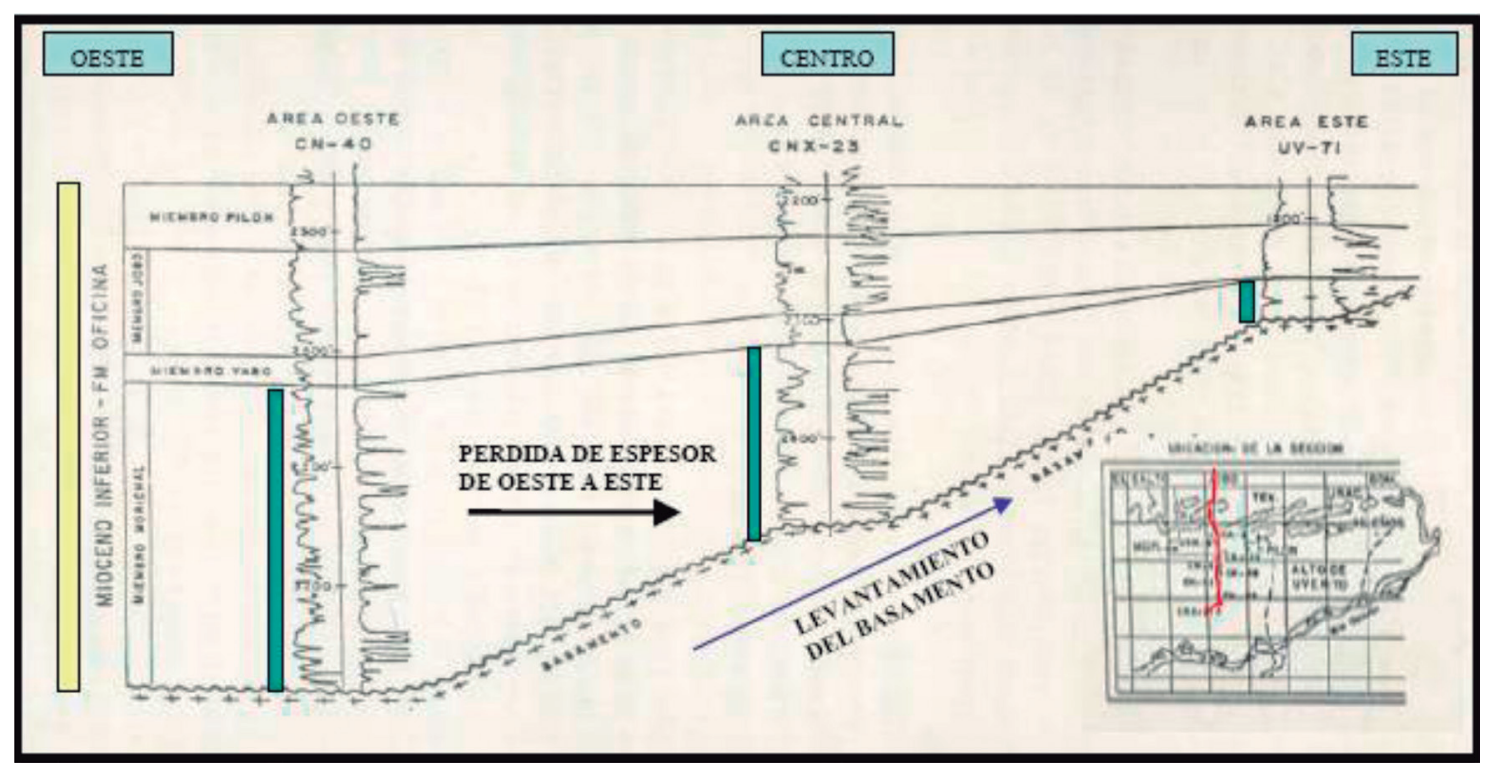

Figura 3. Corte Estructural del Bloque Carabobo (Tomado de Crespo, 2008)

Por lo que la configuración geológica descrita nos refleja que el sistema de retención de hidrocarburos es complejo en el campo a tratar en la investigación desde el punto de vista estratigráfico, ya que es posible ver la presencia de diferentes zonas de arenas con hidrocarburos sin conexión aparente entre sí. La extensión lateral puede estar formada por cuerpos de arenas con hidrocarburos libres cerca de la superficie, que migraron debido a la ausencia de una capa confinante de baja permeabilidad (arcilla o arena arcillosa) a lo largo de todo el bloque en el tope de la secuencia sedimentaria. (Crespo, 2008).

\section{Método}

La interpretación se llevó a cabo mediante los métodos de Mandelbrot, mejor conocidos como métodos fractales con la finalidad de generar pseudopozos de origen fractal para expandir la información de las propiedades de los registros en el campo (Belmares, 2011). Para ello se debe verificar la distribución estadística de estos registros, mediante histogramas de frecuencias, debido a que el método fractal solamente es aplicable en bases de datos con distribución Gaussiana (Mandelbrot, 1977), ya que es la manera de reflejar y reproducir la naturaleza fractal del dato.

Partimos de la determinación del volumen de arcilla, obtenidos de los registros petrofísicos de rayos gama "ecuación (1)", y del de registro de porosidad efectiva "ecuación (2)" que se determina mediante los registros de neutrón, y el del volumen de arcilla calculado previamente.

Vsh $=($ GR Leído - GR min. $) /($ GR Max. - GR min. $)$.

$$
\text { Porosidad Efectiva }=\text { Nphi } \times(1-V s h) \text {. }
$$

Luego de los cálculos se procedió a realizar los histogramas de frecuencia de los datos pertenecientes a los registros de rayos gama, resistividad y porosidad efectiva, con la finalidad de verificar como se indicó previamente la distribución de los datos de cada propiedad, obteniendo que los registros de rayos gama y resistividad presentaron un comportamiento logarítmico en su distribución, mientras que el de porosidad efectiva cumplió con la condición necesaria del método de presentar distribución Gaussiana de los datos.

Verificado el comportamiento de los datos a partir de los histogramas, se corroboró la necesidad de normalizar los datos de los registros de rayos gama y los de resistividad, para así poder reproducir el comportamiento fractal de los datos de los registros. Luego de normalizados se reelaboran los histogramas de frecuencia para verificar la distribución gaussiana de los mismos (Fig. 4). 

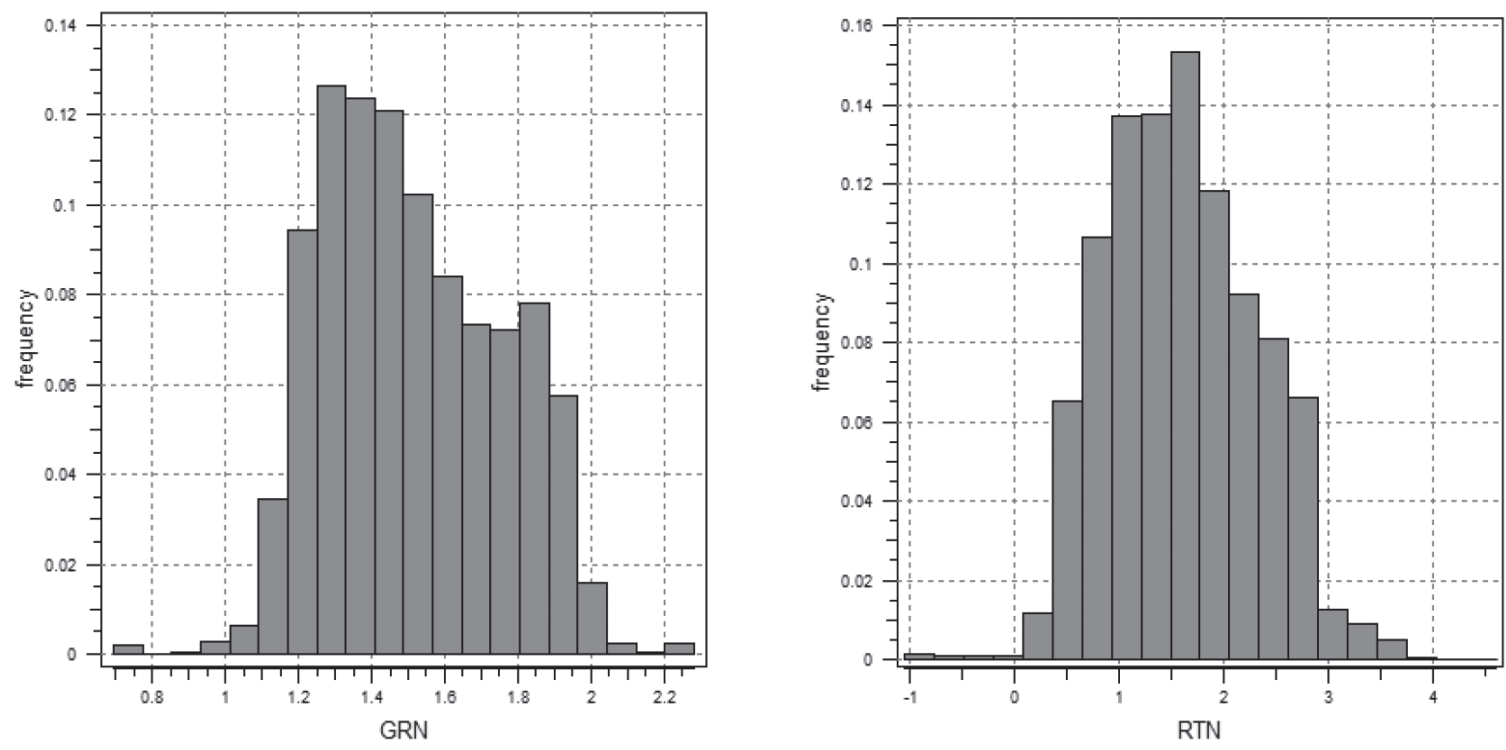

Figura. 4. Histogramas de frecuencia de los registros petrofísicos GR, Rt normalizados.

Normalizados los datos se procedió a la aplicación del método de rango reescalado $(\mathrm{R} / \mathrm{S})$, que no es más que un método estadístico que depende del tamaño de la serie y que se define como el rango de la variación de la serie expresada en términos de su desviación estándar con el fin de estimar el coeficiente de Hurst $(\mathrm{H})$ que corresponde a la pendiente obtenida por un método clásico de regresión lineal ajustado por un estimador de mínimos cuadrados ordinarios y la dimensión fractal de los registros (GR, Rt, Porosidad efectiva). El rango es obtenido de los variogramas experimentales, mientras que la desviación estándar es proveniente del histograma de frecuencia de cada serie (registros petrofísicos). En donde $\mathrm{R}$ es la diferencia entre el máximo y el mínimo de la desviación acumulada y depende del periodo (S) considerado, para poder comparar los rangos diferentes, Hurst reescalo el rango dividiéndolo entre la desviación estándar como mencionamos al inicio del párrafo. Siendo demostrado por Mandelbrot (1968) que el mismo se relaciona con el tamaño de la muestra. Codificado a partir de un algoritmo de re-escalamiento $(\mathrm{R} / \mathrm{S})$ implementado en lenguaje de programación. El cual tiene como fin verificar si la señal generada del reescalamiento es aleatoria "ruido blanco" que se caracteriza por valores de señal de tiempos diferentes que no guardan correlación estadística, esto quiere decir que la señal emitida es una constante. (Fig. 5).

El ruido blanco es indicativo de que le coeficiente de Hurst $(0<\mathrm{H}<1)$ se encuentra por encima de 0.5 , implicando que se puede propagar la propiedad a distancias largas. El coeficiente de Hurst " $\mathrm{H}$ " de una serie es importante porque muestra el grado de rugosidad de los datos, dado que los valores de Hurst son mayores a 0.5 (Fig. 6) es una serie persistente. El mismo se determinó mediante un análisis que se realizó escogiendo cierto número de escalas, con el propósito de obtener la media del vector y calcular las diferencias de cada valor con respecto a la media de los datos (Registros de rayos gamma, resistividad y porosidad efectiva) y hacer una suma acumulativa, basándonos en el análisis estadístico de rango reescalado $(\mathrm{R} / \mathrm{S})$, según Hurst, es proporcional a ctH, donde c es una constante (0.5) y t es la escala en cuestión (Pérez, 1994). Así que el grafico de $\log (\mathrm{R} / \mathrm{S})$ vs t, nos determina el coeficiente de Hurst como la pendiente de la recta.

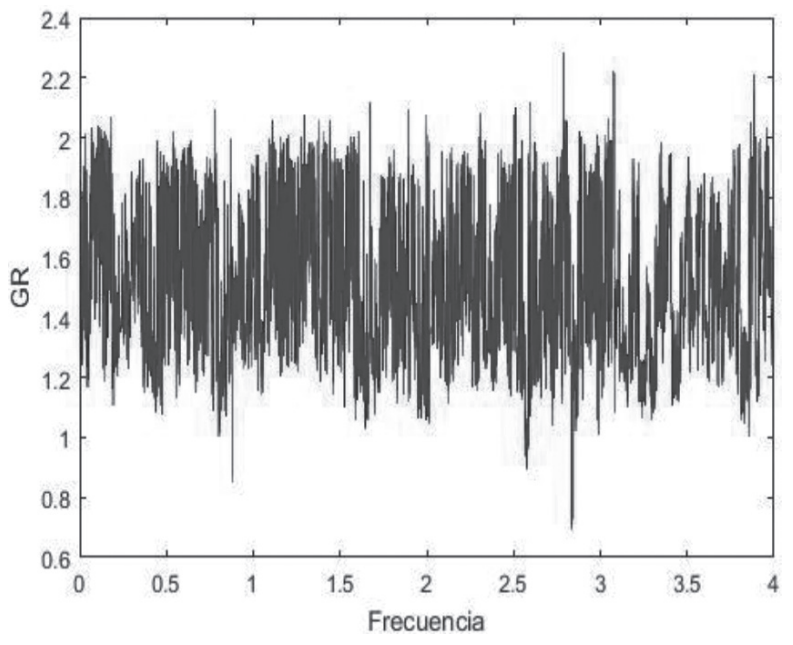

Figura 5. Datos de rayos gama en función de la frecuencia mostrando un comportamiento de ruido blanco. 


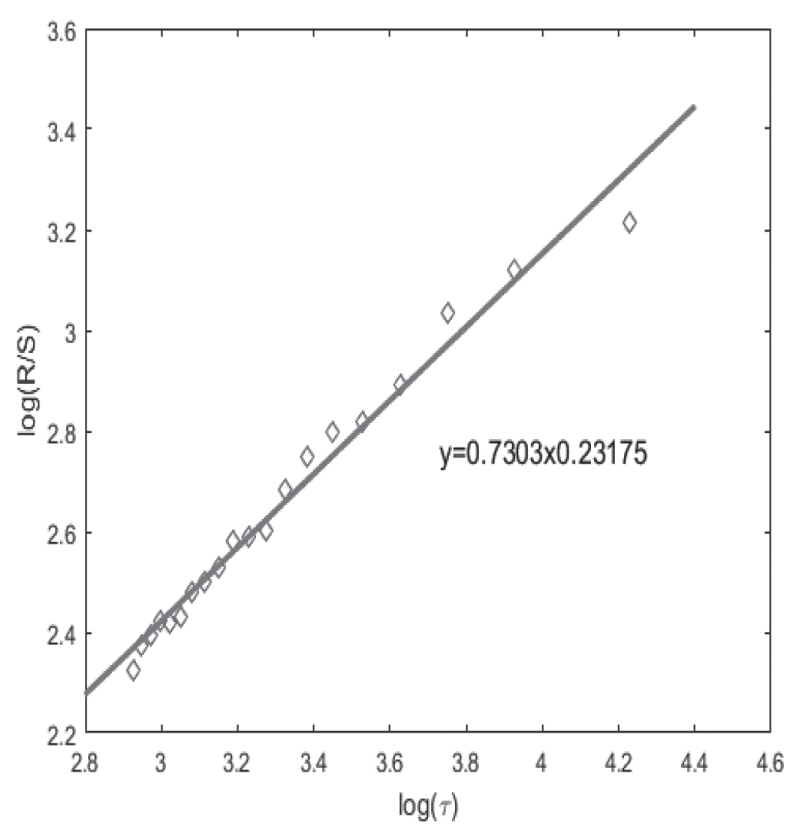

Figura 6. Representa el análisis de rango reescalado, de donde se extrae el exponente de Hurst (la pendiente es el coeficiente de Hurst).

Posteriormente con el método de distribución de parámetros petrofísicos con modelado fractal, el cual consistió en obtener la distribución de cada propiedad petrofísica en los pozos, es decir, se determinaron las profundidades y los valores máximos y mínimos de la ventana común (tabla 1). El siguiente paso consistió en establecer los posibles enlaces entre el conjunto de pozos (Fig. 7).

Tabla 1. Valores de máximos y mínimos de las coordenadas (UTM) y la profundidad de los pozos.

\begin{tabular}{|c|c|c|c|}
\hline Rangos & Coordenada X & Coordenada Y & Profundidad Z \\
\hline Máximos & 506100.0053 & 961930.0003 & 3289 \\
\hline Mínimos & 493679.9987 & 941112.9878 & 286 \\
\hline
\end{tabular}

Luego de establecidos los enlaces entre los pozos y obtenido el coeficiente de Hurst, se realizó el método de adición aleatoria sucesiva (interpolación fractal) mediante el algoritmo modificado de la correlación de series de Mandelbrot por Liu. H (Herrera, 2007), considerando un movimiento browniano, en donde el valor medio del cuadrado de la longitud del desplazamiento experimentado por una partícula es proporcional al tiempo que se invierte en dicho desplazamiento. La generalización del parámetro $0 \leq$ $\mathrm{H} \leq 1$ se denomina movimiento browniano fraccional, donde el desplazamiento de $\mathrm{x}$ son estadísticamente autosimilares respecto al parámetro $\mathrm{H}$, exponente de Hurst. De dichos desplazamientos para los puntos medios de Dn que tiene varianzas $(H=1 / 2)$, generando pseudopozos a partir de los registros existentes.

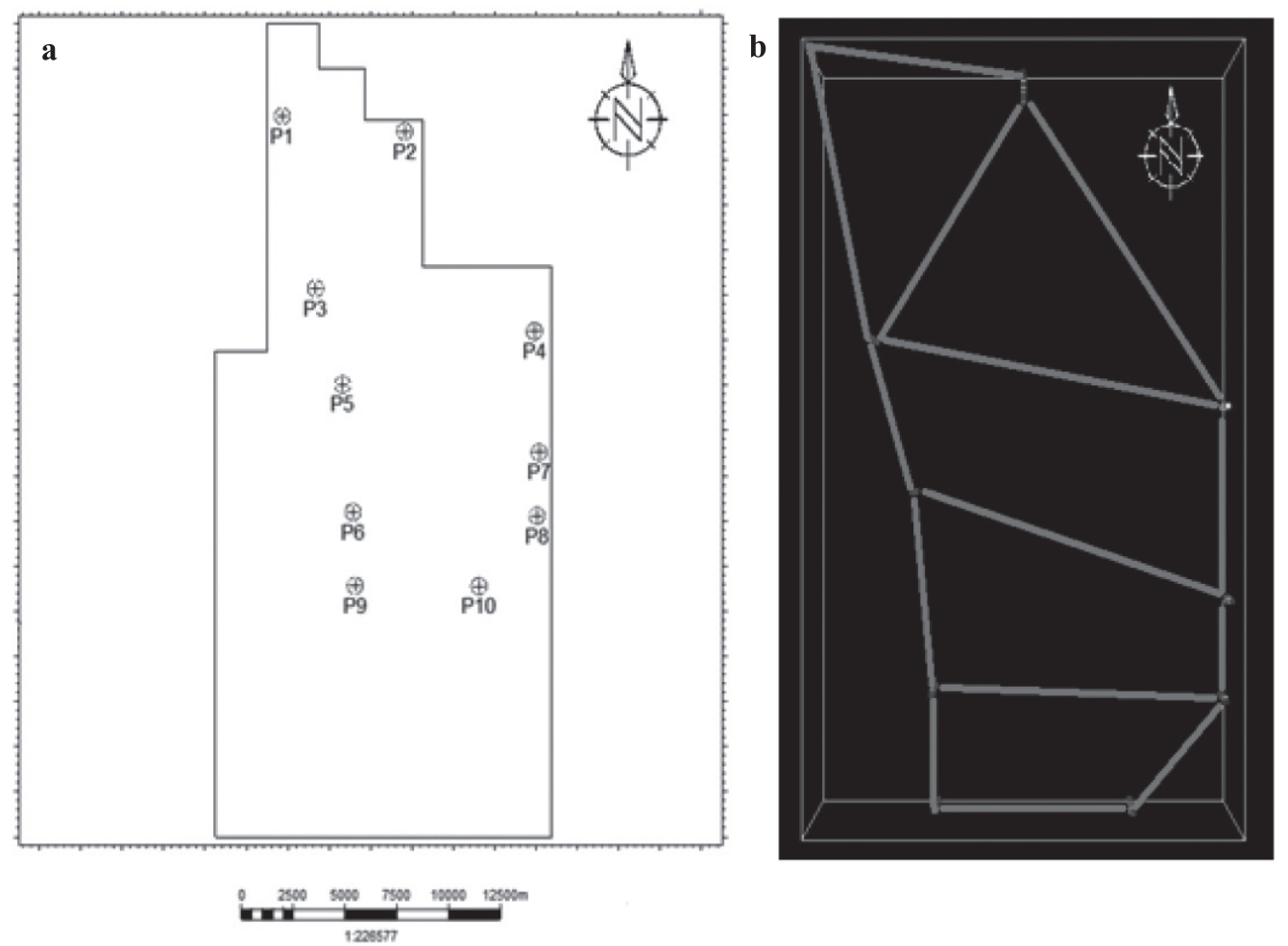

Figura 7. a) localización de pozos originales, b) enlace entre el conjunto de pozos seleccionados (líneas rojas). 
Partiendo de la adición de números aleatorios de la distribución gaussiana $\mathrm{N}\left(0\right.$, Varianza $\left._{0} / 2\right)$ a los dos puntos extremos iniciales, Luego se interpola linealmente el valor del punto medio obtenido a partir del valor de los puntos extremos del paso previo. Posteriormente con tres puntos definidos se vuelve a añadir números aleatorios de la distribución gaussiana $\mathrm{N}\left(0\right.$, Varianza $\left._{1} / 2\right)$ al punto medio generado a partir del segundo paso y a los puntos ya existentes. Luego linealmente se realiza la interpolación entre los tres puntos obtenidos en el paso previo. Y se les añade otro valor aleatorio a los puntos medios interpolados en el paso anterior y a los otros puntos existentes a partir de la distribución gaussiana $\mathrm{N}\left(0\right.$, Varianza $\left._{2} / 2\right)$. Finalmente se repite el procedimiento manteniendo la interpolación lineal y la adición de números aleatorios sucesivos de la distribución gaussiana $\mathrm{N}\left(0\right.$, Varianza $\left._{\mathrm{n}} / 2\right)$ quedando la fórmula de interpolación dominada por el coeficiente de Hurst "Ecuación (3)" como sigue a continuación

$$
\sigma_{\mathrm{n}^{2}}=\left(\sigma_{\mathrm{n}-1^{2}}\right) / 2^{2 \mathrm{H}}=\left(\left(\sigma_{0^{2}}\right) /\left(2^{2 \mathrm{H}}\right)^{\mathrm{n}}\right) *\left(1-\left(2^{2 \mathrm{H}} / 4\right)\right.
$$

Ya con los datos de los pseudopozos y pozos originales juntos (Fig. 8) se procedió a desnormalizar los datos y llevarlos a su escala original.

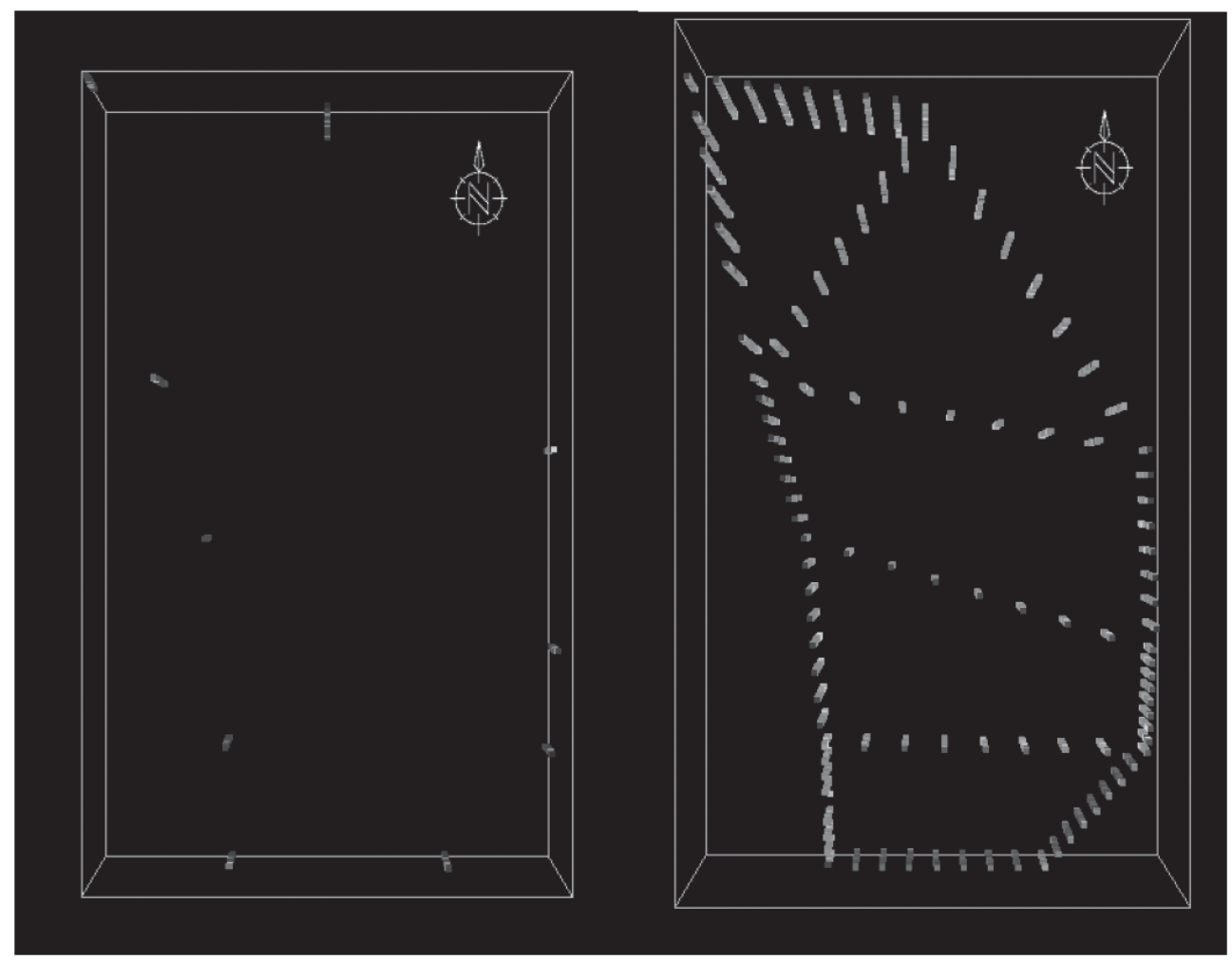

Figura. 8. a) pozos originales en el área de estudio y b) conjunto de pseudopozos generados a partir de modelación fractal con pozos originales en el área de estudio.

Finalmente se calcularon los registros de las propiedades petrofísicas de arena neta "ecuación (4)", arena neta petrolífera "ecuación (5)" y volumen de arcilla "ecuación (1)", para cada uno de los pozos.

$$
\begin{gathered}
\mathrm{AN}=(\mathrm{GR}<=70.0 .3) \\
\mathrm{ANP}=(\mathrm{GR}<=70 \text { and } \mathrm{Rt}>=100.0 .2)
\end{gathered}
$$

La secuencia de procesos posteriores considera el histograma y el modelado del variograma, cuyos parámetros son necesarios para los procesos de estimación y simulación que generan la distribución espacial de las propiedades petrofísicas, llevados a cabo mediante el método estadístico de Kriging.

\section{Resultados y discusión}

Para caracterizar petrofísicamente a la Formación Oficina, considerando los 10 pozos del área de estudio se crearon los pseudopozos mediante la interpolación por modelado fractal, los cuales tenían registros radioactivos de rayos gamma, neutrón y registros eléctricos de resistividad profunda. Con esta muestra, se realizó el 
análisis basándose en los métodos fractales planteados por Mandelbrot (1977) y Remy et al (2009). Creando pseudopozos y propagando las propiedades petrofísicas mediante métodos estadísticos de estimación como el Kriging y de simulación de los mismos. Con la finalidad de proponer un modelo de isopropiedades petrofísica e identificar posibles prospectos exploratorios en la Formación Oficina y compararlos con estudios convencionales, para así verificar la veracidad del método fractal.

Iniciamos con la distribución del registro de porosidad efectiva del área de estudio, en donde se puede distinguir tres zonas bien zonificadas dos de baja porosidad $(0.1 \%$ a $0 \%)$ que se observan al norte y al sur del área y una zona con porosidades económicamente atractivas $(0.2 \%$ a $0.55 \%)$ en la región central a lo largo del espesor de la formación oficina, con algunas ramificaciones bien marcadas con dirección noroeste
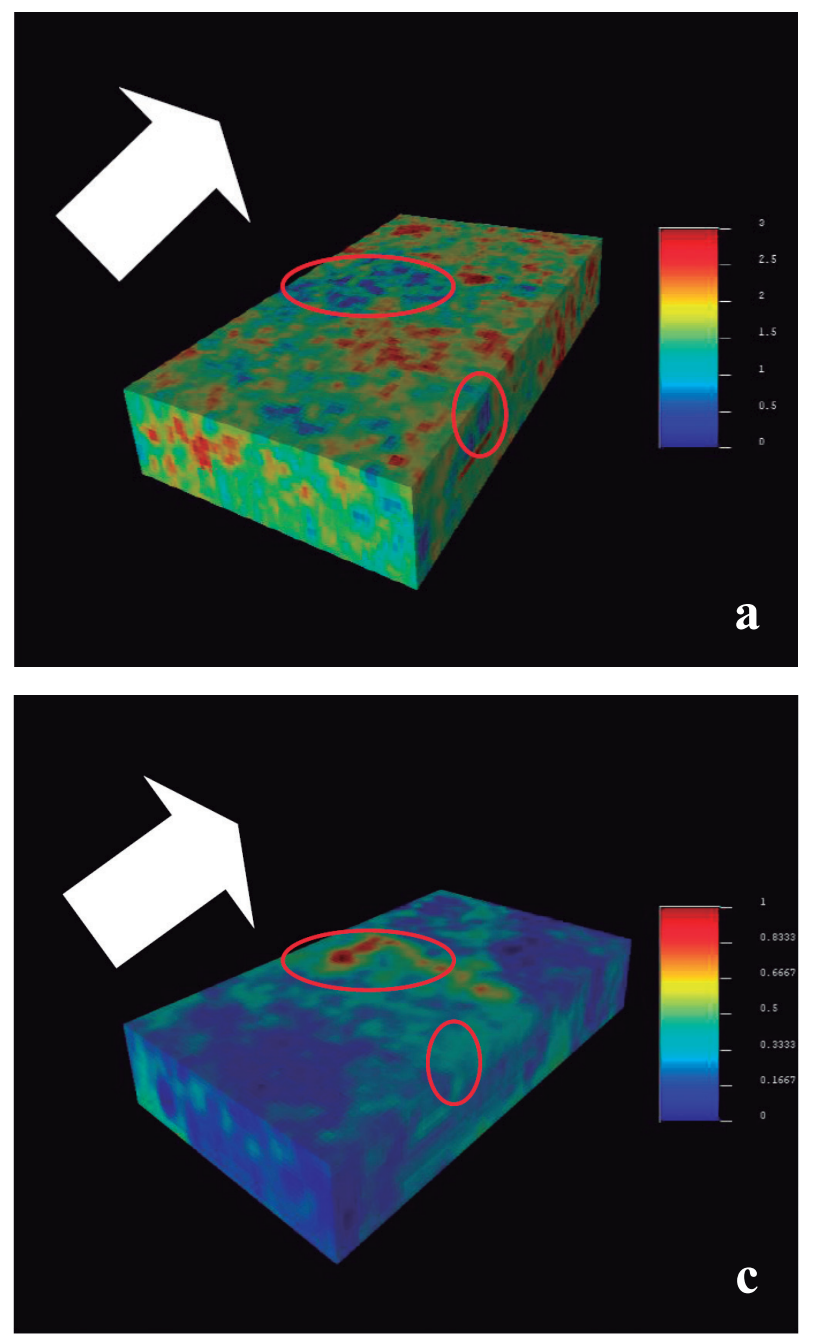

Figura. 9. Distribución especial de propiedades petrofísicas: a) Arena neta (AN), b) Arena Neta Petrolífera (ANP), c) Porosidad efectiva y d) arcillosidad (Vsh) (la flecha blanca indica el norte) (círculos rojos indican prospectos).

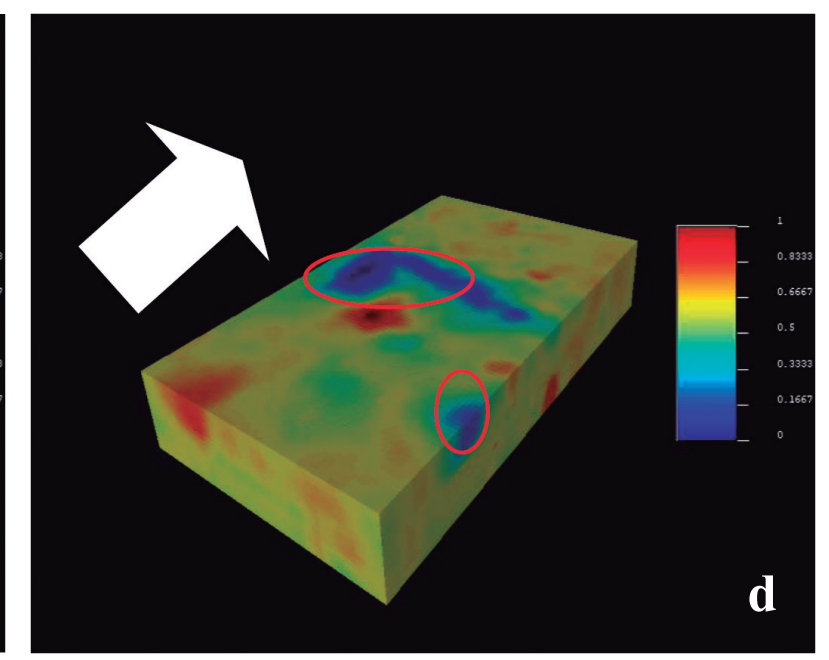

y este-oeste "Fig.9c" y "Fig. 10c". Esto nos permite enfocar nuestra área de interés prospectiva hacia la región central específicamente, que conformaremos o descartaremos con el análisis de la distribución de las otras propiedades.

En la distribución espacial de la propiedad de arcillosidad (Vsh) se observa una tendencia de alta arcillosidad entre las franjas de la zonas sur y norte $(0.6 \%$ a $1 \%)$ siendo muy marcado los valores altos $(0.8 \%$ a $1 \%)$ en la zona suroeste con ramificaciones puntuales al centro del área. En él se distingue un sector de muy baja arcillosidad cerca de $0 \%$ con dirección casi este-oeste en la zona central del área, y arcillosidad de $0.1 \%$ a $0.2 \%$ en la zona sureste superior "Fig. $9 \mathrm{~d}$ " y "Fig. 10d", siendo coincidente con las zonas de alta porosidad, por lo tanto, nos aporta otro elemento para dar más certidumbre sobre la posibilidad del prospecto a definir en el área.

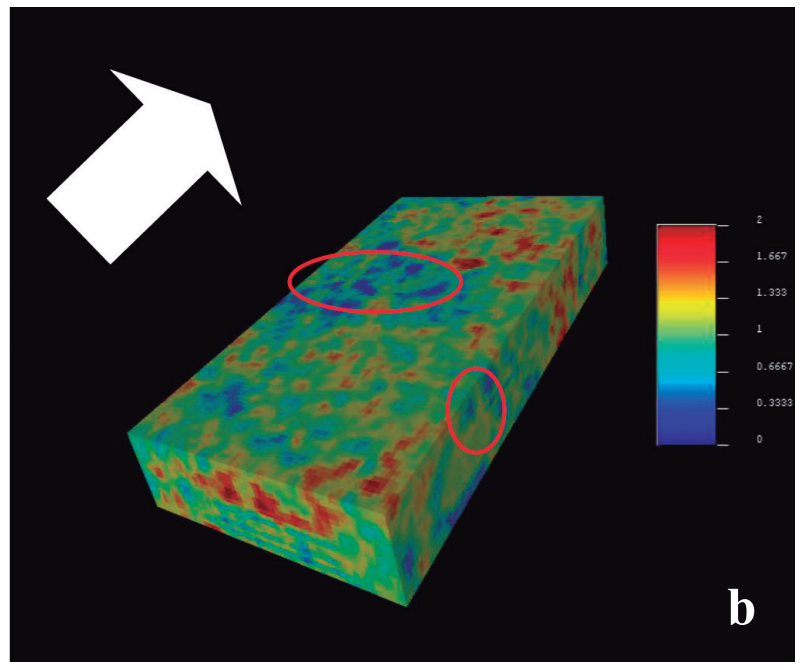


En cuanto a la distribución de arena neta del área de estudio, se observa en la Formación Oficina una tendencia de paquetes de arena de mayor espesor en la zona central con bifurcaciones de canales hacia el sureste y algunos canales con sentido este-oeste en el sur, es decir, el aporte sedimentario proviene del sur sureste hacia el norte noroeste del área de estudio. Siendo este patrón concordante con la información obtenida de las distribuciones de porosidad efectiva y arcillosidad del área, mejorando aun la tendencia a la definición de prospectos en la zona central, ya que presenta altas porosidades $(0.3 \%$ a $0.5 \%)$, baja arcillosidad $(0 \% 0.2 \%)$ y altos espesores de canales de arena limpia, como lo se observa en las "Fig.9a" y "Fig. 10a".
Finalmente, en la distribución espacial de la propiedad petrofísica de arena neta petrolífera se puede distinguir las zonas de arena con presencia de hidrocarburos "Fig.9b" y "Fig. 10b". En ella se observan que las tendencias de los espesores de arena limpia son mantenidas en esta propiedad por lo que podemos afirmar que los espesores de arena limpian encontrados en el área poseen hidrocarburos en ellos, y bajo lo observado en la información de porosidad y arcillosidad antes descrito se puede reconocer ciertas áreas con buena posibilidad para definir prospectos de interés en el campo. A continuación, se muestran las zonas seleccionadas como prospectivas en las cuatro propiedades dentro de las "Fig.8" y "Fig. 9", con el fin de detallar las áreas con mayor factibilidad de albergar hidrocarburos.


Figura. 10. Distribución especial de propiedades petrofísicas: a) Arena neta (AN), b) Arena Neta Petrolífera (ANP), c) Porosidad efectiva y d) arcillosidad (Vsh) (la flecha blanca indica el norte) (círculos rojos indican prospectos).

\section{Comparación con métodos convencionales}

En las distribuciones espaciales de las propiedades de las porosidades efectivas (Fig. 11) del área, se distingue claramente un comportamiento similar con ambos métodos que corroboran la tendencia de las porosidades altas en la región noroeste central del campo. Pero en el modelo estadístico fractal se observa una mejora en la interconectividad de los poros, ya que podemos seguir la interconexión poral hacia el sur, que no podemos observar en los métodos convencionales. Así que el volumen del método fractal está más cerca del comportamiento real de la propiedad. 




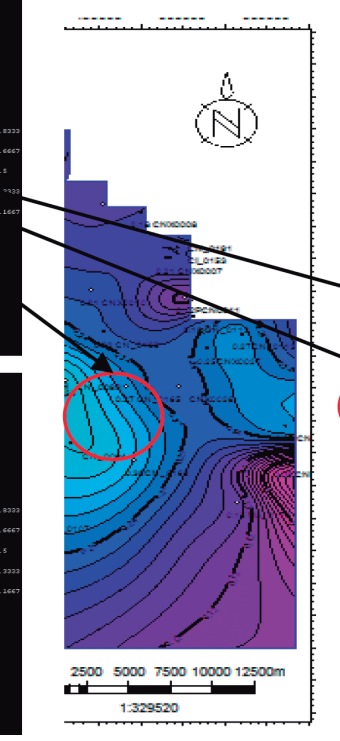

a

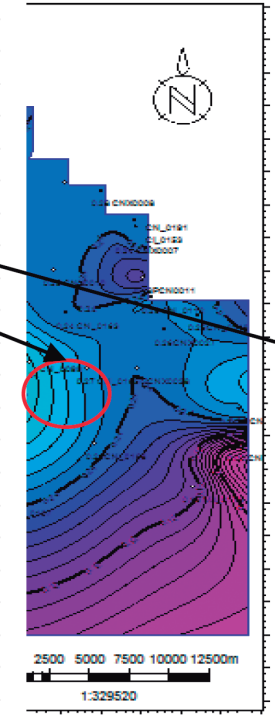

b



$\mathrm{c}$

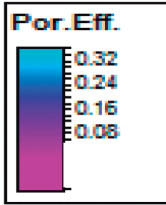

Figura 11. Distribución espacial de las propiedades petrofísicas de la porosidad efectiva (método fractal) en comparación con los mapas de porosidad efectiva por el método convencional de la Formación Oficina (a) Morichal superior, b) Medio c) inferior respectivamente), (blanco flecha que apunta al norte) (círculos rojos definieron prospectos).
Teniendo en cuenta los mapas de arena neta de petrolífera obtenidos de las propiedades petrofísicas generadas por los métodos convencionales de la Formación de Oficinas y comparándolos con los volúmenes creados por los métodos fractales de la misma propiedad, se corrobora que ambos métodos nos permitieron definir las mismas perspectivas de interés. Aunque observa claramente cómo con los métodos fractales, las tendencias de los mismos se definen con mayor claridad tanto horizontal como verticalmente. Por lo que el método fractal es extremadamente útil porque permite una mejor definición de las perspectivas, observe que en ambos métodos se distinguen las zonas de arena con hidrocarburos (Fig. 12), pero al igual que con las propiedades mencionadas anteriormente, con el método estadístico fractal es posible establecer un mejor rastreo de las arenas con hidrocarburos en la formación.


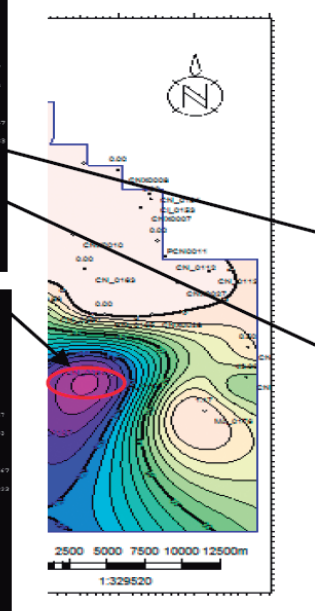

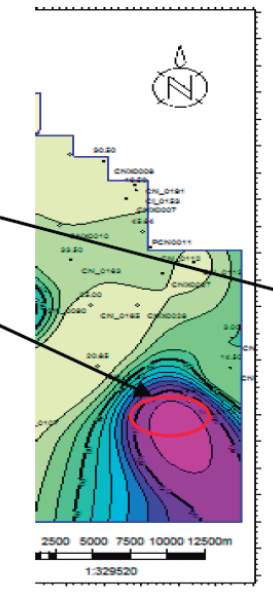

b

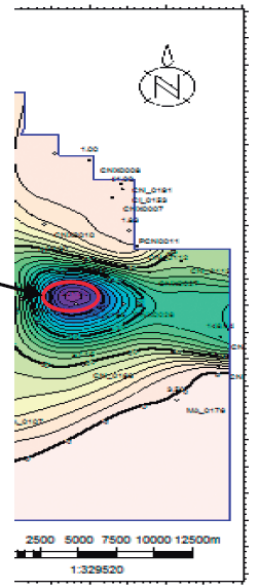

Figura 12. Distribución espacial de las propiedades petrofísicas de Arena Neta Petrolífera (método fractal) en comparación con los mapas de porosidad efectiva por el método convencional de la Formación Oficina (a) Morichal superior, b) Medio c) inferior respectivamente), (blanco flecha que apunta al norte) (círculos rojos definieron prospectos). 


\section{Conclusiones}

Se corroboro que los métodos fractales utilizados a partir del análisis estadístico de kriging ordinario y simulación gaussiana fueron una herramienta de gran ayuda en la estimación de propiedades petrofísicas debido a la naturaleza fractal del dato. En cuanto a los parámetros estadísticos utilizados se pudo verificar que el rango de varianza fue determinante en la interpolación lineal de los datos en base a la distribución y alcance espacial de los mismos.

El uso de la variación del método de adición aleatoria sucesiva de Mandelbrot presentado por Liu, logro establecer un modelado de registros de pozos de manera efectiva, debido a que presentan tendencias periódicas y aleatorias. Gracias a aleatoriedad se pudo caracterizar en términos del coeficiente de Hurst y a partir de él se modelaron los pseudo pozos de origen fractal.

Del modelado fractal podemos afirmar en base a los resultados que es una herramienta complementaria a los métodos convencionales de gran utilidad y eficacia en la caracterización de petrofísica de los yacimientos. En cuanto a posibles prospectos en la Formación Oficina en el campo en la región central y hacia el sureste, lo cual corrobora la información previa obtenida de los análisis con métodos convencionales. Pero además mejoro la visualización de la interconexión poral y sus ramificaciones en profundidad y hacia el sur, que no logran verse con los mapas de métodos convencionales, de tal manera que se puede vislumbrar un aumentando de las capacidades de almacenamiento de hidrocarburos por parte de esas rocas.

Finalmente, El análisis conjunto de las propiedades presentadas en esta investigación permite dilucidar con un mayor asertividad la ubicación y extensión laterales y verticales de las perspectivas de interés, que con los métodos convencionales es más complejo de delimitar.

\section{Bibliografía}

1. Banks, L.M (1956). Historia estructural y yacimientos petrolíferos del centro de Anzoátegui. Acta Cient. Venezol., edición 7 (8): pp 176-184.

2. Belmares, J (2011). Distribución especial de parámetros petrofísicos por modelación fractal en la caracterización estática de yacimientos. Trabajo Especial de Grado. Instituto Politécnico Nacional.

3. Busnego H. (2012). Generación de un modelo petrofísico y análisis de incertidumbre de las variables de roca y fluído, relacionadas con los parámetros de corte calibrados con información de núcleos pertenecientes al bloque 5 , área petroindependencia, división Carabobo de la faja petrolífera del orinoco. Tesis Especial de Grado. Universidad Central de Venezuela, Caracas.

4. Calvo, Arturo. (2008). Possibilities to increase the Recovery Factor in Petromonagas area, Orinoco Heavy Oil Belt, Venezuela. Trabajo Especial de Grado, The Robert Gordon University, Aberdeen School of Engineering, pp. 6-17.

5. Chramcow, D. (2008). Interpretación Sismoestratigráfico del área de Pichincha perteneciente a la Faja Petrolífera del Orinoco. Informe final de cursos en cooperación. Universidad Simón Bolívar, Caracas.

6. Crespo, J. (2008). Modelo sedimentológicoestratigráfico, bloque Carabobo 2, Faja Petrolífera del Orinoco. Trabajo especial de grado. Universidad Central de Venezuela.

7. Escobar, F. H., Cantillo, J. H., \& Santos, N. S. (2011). A practical approach for estimation of the average reservoir pressure from multi-rate tests in long horizontal wells. Revista Fuentes, El Reventón Energético, 9(1).

8. Funkhouser, H.J., Sass, L.S y Hedberg (1948). Santa Ana, San Joaquin, Guario, and Santa Rosa oilfields (Anaco fields), central Anzoátegui, Venezuela. Am. Assoc. Petr. Geol. Bull., edición 32 (10). pp. 1851-1908.

9. Hedberg, H.D. (1950). Geology of the Eastern of Venezuela Basin (Anzoategui-Monagas-SucreEastern Guarico portion). Am. Assoc. Petr. Geol. Bull., edición 48 (11). pp. 1755-1803.

10. Herrera, V. (2007). Simulación numérica de Paisajes montañosos, cuencas hidrográficas y procesos de lluvia, erosión y depositación. Trabajo Especial de Grado. Universidad Simón Bolívar, Sarteneja-Caracas.

11. Mandelbrot Benoit B. (1977). The Fractal Geometry of Nature. New York, USA: W.H Freeman and Company.

12. Mandelbrot and Van Ness. (1968). Fractional browian motion, fractional noise and application, SIAM Review, pp. 442-447.

13. Martín, C. A. G., García, R. E. P., Niño, J. C. L., \& Lombana, H. B. (2014). Optimización de la metodología para el cálculo de porosidad a través de saturación de fluidos. Revista Fuentes, 12(2).

14. Meneses, A. F. O., Moreno, L. F. C., \& Plata, J. A. R. (2017). Metodología experimental para la estimación de permeabilidades relativas en 
dos y tres fases por medio de ajuste histórico. Revista Fuentes, 15(1), 75-85.

15. Meneses, A. F. O., Otero, E. H., \& Santos, N. S. (2017). Estimación de saturaciones in-situ durante experimentos de inyección de fluidos usando tomografía computarizada de rayos X. Revista Fuentes, 15(2), 107-116.

16. Otero, E. H. H. (2016). Generación del modelo geoestadístico de los depósitos fluviales para la formación mugrosa del campo colorado. Revista Fuentes, 14(2), 29-39.

17. Pérez, R. (1994). Programa en Matlab para el cálculo del exponente de Hurst y dimensión fractal de series de tiempo. Recovered 15 of august of 2017, by http://www.geociencias.unam. $\mathrm{mx} / \sim$ bole/eboletin/progHurstyD1108.pdf
18. Remy Nicolas, Boucher Alexander and Wu Jianbing (2009). Geostatistics with SGemS. New York: Cambridge, University Press.

19. Velandia, J. L. P. (2013). Simulación numérica del flujo bifásico agua-petróleo en un medio poroso. Fuentes: El reventón energético, 11(2), 10.

20. Villar, A., Moreno, L. F. C., Hernandez, J. H. C., \& Molina, J. J. R. (2017). Predicción del daño a la formación por acumulación de $\mathrm{CaCO} 3$ durante el flujo constante de salmueras en el medio poroso. Fuentes: El reventón energético, 15(1), 49-57.

21. Yoris, F., \& Ostos, M. (1997). Geología de Venezuela: Geología general y cuencas petrolíferas. Texas: Jolley Printing.

Recepción: 22 de octubre de 2018

Aceptación: 18 de mayo de 2019 